\title{
Distinct evolution process among type I interferon in mammals
}

\author{
Lei $\mathrm{Xu}^{1,2}$, Limin Yang ${ }^{1 凶}$, Wenjun Liu ${ }^{1,2,3 凶}$ \\ ${ }^{1}$ CAS Key Laboratory of Pathogenic Microbiology and Immunology, Institute of Microbiology, Chinese Academy of Sciences, \\ Beijing 100101, China \\ ${ }^{2}$ University of Chinese Academy of Sciences, Beijing 100049, China \\ ${ }^{3}$ China-Japan Joint Laboratory of Molecular Immunology and Molecular Microbiology, Institute of Microbiology, Chinese Academy \\ of Sciences, Beijing 100101, China \\ $\triangle$ Correspondence: liuwj@im.ac.cn (W. Liu), Imyang@im.ac.cn (L. Yang) \\ Received March 12, 2013 Accepted April 8, 2013
}

\begin{abstract}
Interferon (IFN) is thought to play an important role in the vertebrate immune system, but systemic knowledge of IFN evolution has yet to be elucidated. To evaluate the phylogenic distribution and evolutionary history of type I IFNs, 13genomes were searched using BLASTn program, and a phylogenetic tree of vertebrate type I IFNs was constructed. In the present study, an IFNס-like gene in the human genome was identified, refuting the concept that humans have no IFNס genes, and other mammalian IFN genes were also identified. In the phylogenetic tree, the mammalian IFN $\beta$, IFN $\varepsilon$, and IFNK formed a clade separate from the other mammalian type I IFNs, while piscine and avian IFNs formed distinct clades. Based on this phylogenetic analysis and the various characteristics of type I IFNs, the evolutionary history of type I IFNs was further evaluated. Our data indicate that an ancestral IFNa-like gene forms a core from which new IFNs divided during vertebrate evolution. In addition, the data suggest how the other type I IFNs evolved from IFNa and shaped the complex type I IFN system. The promoters of type I IFNs were conserved among different mammals, as well as their genic regions. However, the intergenic regions of type I IFN clusters were not conserved among different mammals, demonstrating a high selection pressure upon type I IFNs during their evolution.
\end{abstract}

KEYWORDS type I IFN, evolutionary history, vertebrate, gene cluster

\section{INTRODUCTION}

Interferon was first recognized half a century ago for its antiviral activities (Isaacs and Lindenmann, 1957), and its anti-proliferation and immune-regulatory activities were then subsequently discovered (Stark et al., 1998). According to the receptors that they bind, IFNs can be divided into three types: type I, type II, and type III IFNs (Sheppard et al., 2003). There are many different kinds of type I IFNs, such as IFNa, $\beta, \delta, \varepsilon, \zeta, \mathrm{K}$, T, and $\omega$, but type II and type III IFNs are only by a single kind each, IFNy and IFN $\lambda$, respectively (Pestka et al., 2004).

Type I IFNs predominantly function through the typical JakStat pathway. When type I IFNs bind to their high-affinity receptors, a heterotrimer named interferon-stimulated gene factor 3 (ISGF3) is formed to activate the expression of interferonstimulated gene (ISGs). Aside from the canonical Jak-Stat pathway, IFNs can also activate the MAPK pathway (Stancato et al., 1997; David, 2002). However, due to its complex activities in the immune system, new IFN pathways and roles remain to be discovered.

Type I IFNs have been identified in zebrafish, Atlantic salmon, grass carp, and Fugurubripes, which several of them display similar activities to their mammal homologs (Altmann et al., 2003; Robertsen et al., 2003). IFN $\beta$ is hypothesized to have arisen from birds, and the other type I IFNs are present only in mammals (Sick et al., 1996).IFNל, also known as limitin, was discovered for its ability to arrest the growth of or kill lympho-hematopoietic cells, and is thought to only exist in mice (Oritani et al., 2000). IFNס has been described in sheep, pigs, and horses and is expressed at 15 days of gestation in conceptus (Lefèvre and Boulay, 1993; Cochet et al., 2009). IFNT is considered to only exist in ruminants but is present in the human genome as a pseudogene (Whaley et al., 1994). Amphibian type I IFN genes were inferred as intron-containing and 4-cysteine-containing IFN genes, similar to zebrafish IFNb and IFNc, while reptile type I IFNs were inferred from genomic se- 
quence as intronless forms (Sun et al., 2009; Qi et al., 2010).

Robert has hypothesized a good model for type I IFN evolution, which suggests that IFN $\omega$ diverged from IFNa and that IFNT arose from IFN $\omega$ approximately 36 million years ago (Roberts et al., 1998). The evolution of IFNais well described, and both gene conversion and duplication played important roles in the formation of the IFNa gene family (Woelk et al., 2007). However, the detailed phylogenic distribution and evolutionary history of type I IFNs in vertebrates remains unclear (Qi et al., 2010).Towards a better understanding of type I IFN evolution, we report several novel IFNs and illustrated the evolution history of type I IFNs in vertebrates. The gene synteny analysis demonstrates a high selection pressure upon type I IFNs during their evolution.

\section{RESULTS}

\section{Type I IFN gene clusters in genomes}

Different types IFN genes are located on different chromosomes. In humans, type I IFN genes represent a gene cluster on chromosome 9 that includes 14 IFNa genes, one IFN $\omega$ gene, one putative IFNס gene, one IFNT pseudogene, one IFN $\beta$ gene and one IFN $\varepsilon$ gene. The IFNk gene is not adjacent to this gene cluster, and a similar situation was found in other mammals. In mouse, type I IFNs form a gene cluster on chromosome 4, which includes 16 IFNa genes, one IFN $\omega$ pseudogene, 14 limitingenes, and one IFN $\varepsilon$ gene. In most species, the IFN $\varepsilon$ and IFN $\beta$ genes are located on the two extremities of type I IFN clusters, while the other type I IFN genes were randomly located relative to one another, except limitin, which forms a small cluster within the type I IFN cluster.

As indicated by the BLAST results, IFNס and IFN $\omega$ genes may not exist in the mouse and dog genomes and this result in not due to lack of sequence coverage as the mouse and dog genomes have been fully characterized (Fig. 1), which is consistent with previous studies (Hardy et al., 2004). Limitins only exist in mice. Indeed, even in the rat genome, the limitin homolog is a pseudogene with an early stop codon and considerably degraded sequence (date not shown).

A putative IFNס gene, containing a pseudogene named IFNa12p, was identified in the human genome (Fig. 2). This gene is located in the type I IFN gene cluster and represents a single gene that is quite different from the other IFNס genes in sheep and pigs. Sharing $62.3 \%$ similarity with horse IFNס1 and $<50 \%$ similarity with the other human IFNs, the putative human IFNס gene is likely a new subtype of human type I IFN (Table 1). Located immediately beside the IFNס gene lies the IFNa8 gene and IFNa11p, which is unexpressed in humans (Henco et al., 1985). In addition, the homolog of human pseudogene in other primates is similar with itself, represents as a single gene with an early stop codon (date not shown).

Four IFN $\varepsilon$ genes were identified in the cow, horse, cat, and dog genomes (Fig. 2A). All of these genes display $>75 \%$ similarity with human IFN $\varepsilon$ and $<50 \%$ similarity with the other IFNs previously identified in these genomes. Similar to human $\operatorname{IFN} \varepsilon$, these four IFN $\varepsilon$ genes are located on the extremities of the type I IFN clusters, i.e., there is high conservation of the IFN $\varepsilon$ genomic location. In addition, three IFNk genes were identified in the horse, cat, and dog genomes (Fig. 2B). Both the phylogenetic tree and sequence similarity indicate that these genes are true IFNK genes. All of these genes are located outside of the type I IFN gene cluster, just like the human IFNk gene.

\section{The complex relationship among type I IFNs in vertebrates}

Within the type I IFN grouping, avian type I IFNs forms a separate clade from piscine and mammalian type I IFNs (Fig. 3). In our analysis, piscine type I IFNs are the outgroup of avian and mammalian type I IFNs, consistent with the course of vertebrate evolution. Unique to piscine IFNs, the divergence pattern of type I IFNs does not match the speciation of fish, and this

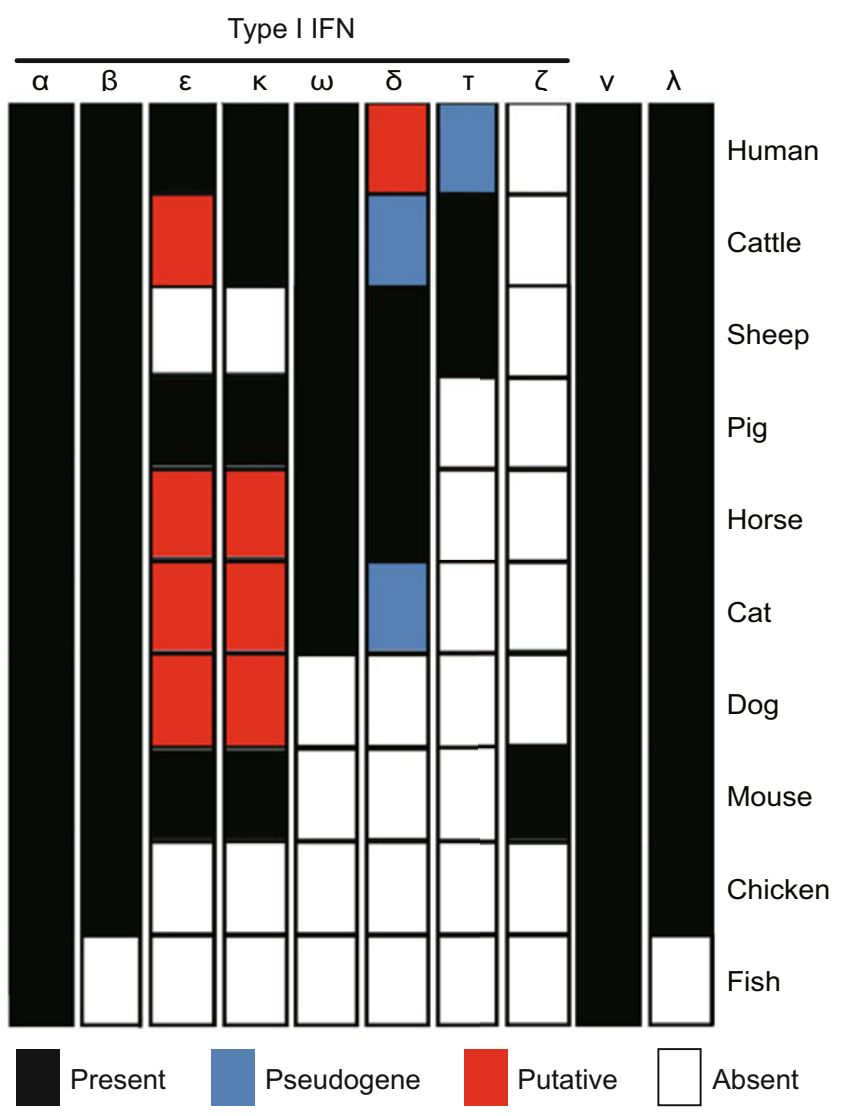

Figure 1. Absence/presence plots in a subset of 10 vertebrate genomes. Type II IFN and IFNa exist in all vertebrates, while type III IFN is absent in fish genomes. IFN $\beta$ can be found in both avian and mammalian genomes, and the other type I IFN only exist in mammal. Mouse and dog lost their IFN $\omega$ genes and IFNס genes during species evolution, but limitins arise in mouse genome. 4 IFN $\varepsilon$ genes and IFNK genes was inferred, suggesting that IFN $\varepsilon$ and IFNK exist in all mammals. IFNT only exist in ruminants. 
A

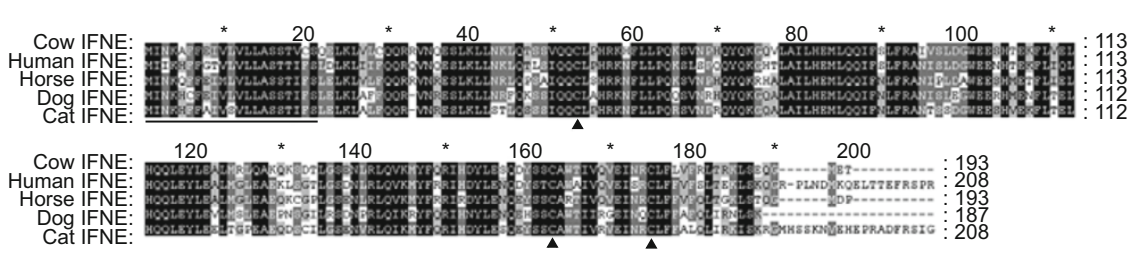

B

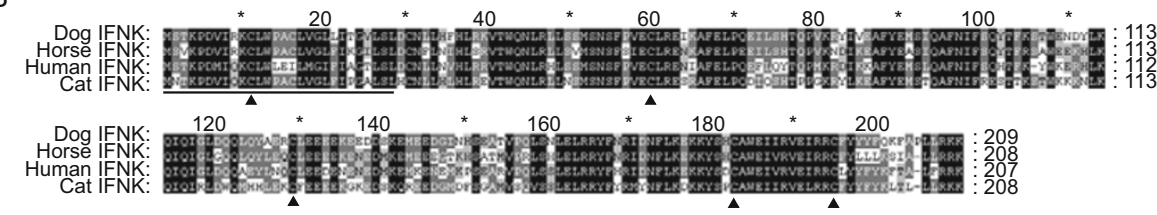

C

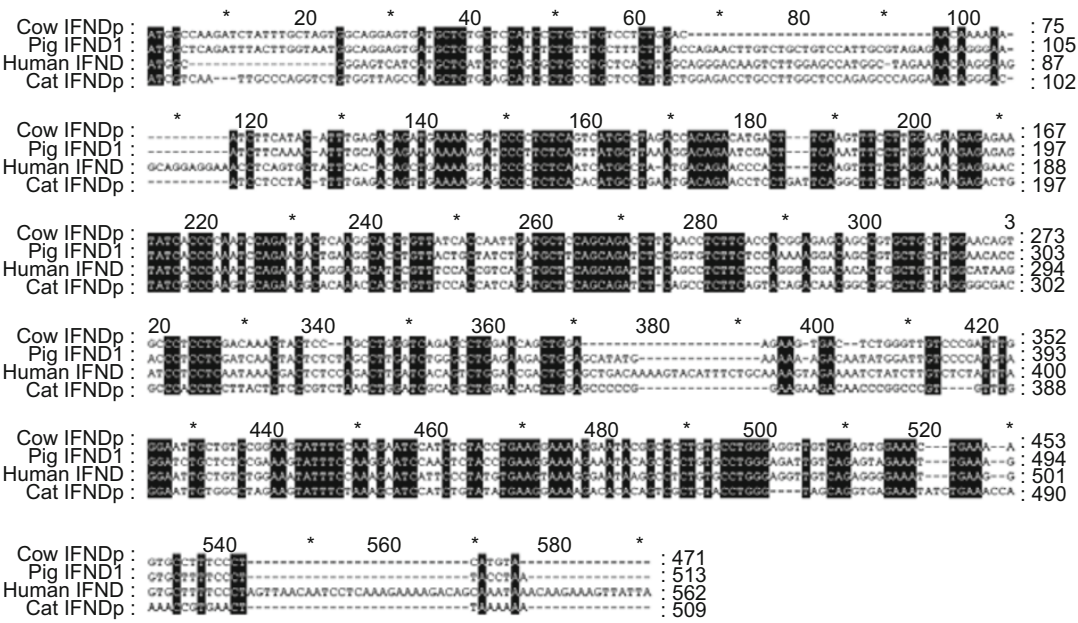

Figure 2. Multiple alignment and gene synteny analysis of novel identified IFNs from human, cat, horse, cow and pig. The alignment was generated by CLUSTALW and then edited by GENEDOC. (A and B) Multiple alignment of novel identified IFN $\varepsilon$ and IFNks. The putative signal peptide sequences predicted by SignalP4.0 are underlined and conserved cysteines are marked with black trilateral. Conserved regions are shadowed. (C) Multiple alignment of novel identified IFNס pseudogene.

disparity was not resolved by restricting the list of piscine type I IFN species in the phylogenetic analyses. Thus, the reason for this discrepancy is the divergence of $2 \mathrm{C}$-containing IFNs and $4 \mathrm{C}$-containing IFNs in fish.

The avian and mammalian IFNs form distinct speciesspecific clades in the phylogenic tree (as the piscine IFNs do). Even though IFNs have been purified from reptiles and have similar biophysical properties as mammalian IFNs, the failure to clone these IFNs makes it difficult to further study reptile type I IFNs.

The major subgroups of IFNs identified thus far form subgroups within the mammalian type I IFN family. Phylogenetically, the mammalian type I IFN subtypes form clades consistent with mammalian speciation in the phylogenetic tree. The first diverging group of IFNs within the mammalian type I IFN clade is that of the unduplicated IFNs. IFNk forms an outgroup in this subclade, which indicates a different evolutionary route compared to IFN $\beta$ and IFNع. The next subgroup to diverge from the remaining mammalian type I IFNs is that of IFN $\varepsilon$ and IFN $\beta$. These two subgroups may in fact be more related to each other than to other mammalian type I IFN subtypes.

Both IFNס and IFNל form an outgroup from the remaining mammalian IFNs. Like the piscine IFNs, the divergence pattern of porcine IFNס genes does not form a single clade in the phylogenetic tree, suggesting a close evolutionary distance among IFNס genes from different species. The failure to find limitin in the pig and horse genomes, which have several IFNס genes, and the failure to find IFNס genes in mice and rats, suggests a preferential evolutionary relationship between IFNס and IFN $\zeta$.

IFN $\omega$ and IFNT form a subgroup in the phylogenetic tree, and porcine and bovine IFN $\omega$, together with ovine and bovine IFNT, form a core clade in this subgroup. Though IFN $\omega$ and IFNT display intimate relationships, their functions are quite different: IFN $\omega$ is an antiviral and immuno-regulator, just like IFNa, while the preferential function of IFNT is to ensure the pregnancy continues through preventing the corpus luteum from degradation. Aside from their differences in function, the species distribution of IFN $\omega$ is more extensive than that of IFNT, which is only found in sheep and cattle. According to the phylogenetic tree and the characteristics of IFN $\omega$ and IFNT, 
Table 1. Sequence identities and divergences among type I IFNs

\begin{tabular}{|c|c|c|c|c|c|c|c|c|c|c|c|}
\hline & CalfA1 & EqcA1 & FecA1 & HosA1 & BotB & CalfB & FecB & EqcB & HosB & EqcD1 & HosE \\
\hline CalfA1 & - & 69.5 & 73.2 & 65.2 & 32.3 & 35.8 & 39 & 38.7 & 32.8 & 44 & 36.2 \\
\hline EqcA1 & 30.3 & - & 73.5 & 79.8 & 34.2 & 37.3 & 39.1 & 40.7 & 39.6 & 51.8 & 40 \\
\hline FecA1 & 25.4 & 22.9 & - & 69.8 & 35.1 & 36.2 & 37.8 & 38.5 & 34.4 & 46.6 & 36.8 \\
\hline HosA1 & 36.4 & 19.6 & 27.3 & - & 36.4 & 36.4 & 39.2 & 40.5 & 36.5 & 52.6 & 42.5 \\
\hline BotB & 93.6 & 88.1 & 87.8 & 91.1 & - & 65.8 & 67 & 70.1 & 65.4 & 33.7 & 39.6 \\
\hline CalfB & 85.6 & 85 & 88.4 & 88.6 & 38.6 & - & 82 & 74.2 & 71.5 & 36.7 & 41 \\
\hline $\mathrm{FecB}$ & 79.2 & 77.9 & 79.4 & 81.5 & 35.4 & 17.5 & - & 75.4 & 71.7 & 34.5 & 41.2 \\
\hline EqcB & 77.8 & 73.5 & 76.2 & 77.9 & 31.8 & 28.4 & 25.2 & - & 73.8 & 37.3 & 40.5 \\
\hline HosB & 87.3 & 80.6 & 90 & 86.5 & 36.8 & 30.2 & 28.7 & 27.6 & - & 34.7 & 43.1 \\
\hline EqcD1 & 65.8 & 56.1 & 62.6 & 55.7 & 92.1 & 86.7 & 94.1 & 84.5 & 86.1 & - & 38.3 \\
\hline HosE & 90.5 & 81 & 88 & 73.8 & 87.3 & 81.1 & 82.8 & 76.1 & 77.3 & 90.1 & - \\
\hline EqcW1 & 51.4 & 41.6 & 47.5 & 41.8 & 83.7 & 78.4 & 74.3 & 68.4 & 69.1 & 58.1 & 75.2 \\
\hline FecW1 & 53.1 & 47.7 & 51.6 & 50.3 & 91.3 & 89.3 & 83.4 & 79.6 & 87.6 & 65.6 & 93.2 \\
\hline HosW & 51.2 & 39.1 & 42.3 & 38.9 & 79.3 & 74 & 72.2 & 64.4 & 73.7 & 52.4 & 77.8 \\
\hline HosT & 45.8 & 36.9 & 39.4 & 37.5 & 83.4 & 77.3 & 75.6 & 66.6 & 76.6 & 59 & 75 \\
\hline FecDp & 86 & 71.1 & 83.2 & 71.9 & 87.8 & 82 & 91.1 & 85 & 98.4 & 30.6 & 82.9 \\
\hline HosD & 79.7 & 71.3 & 75.5 & 63.2 & 89.5 & 86.9 & 96.4 & 90 & 94.4 & 28.9 & 96.1 \\
\hline CalfE & 91.3 & 76.4 & 89.2 & 77.5 & 92.2 & 88.1 & 85.1 & 83.6 & 80.7 & 88.6 & 19.6 \\
\hline CalfK & 126.8 & 111.3 & 122.1 & 101.8 & 144.3 & 124.2 & 127.9 & 136.8 & 123.1 & 129.8 & 108.1 \\
\hline EqcE & 96.7 & 82.8 & 91.1 & 79.3 & 84.3 & 79.1 & 81.4 & 75.2 & 78.3 & 91 & 16.7 \\
\hline EqcK & 122.6 & 110.1 & 117.6 & 105.3 & 122 & 116.5 & 112.1 & 119.8 & 114.4 & 132.7 & 107.2 \\
\hline \multirow[t]{2}{*}{ FecE } & 92.5 & 81.2 & 91.2 & 81.1 & 91.2 & 83.3 & 81.9 & 80.2 & 77.4 & 89.7 & 22.1 \\
\hline & EqcW1 & FecW1 & HosW & HosT & FecDp & HosD & CalfE & CalfK & EqcE & EqcK & FecE \\
\hline CalfA1 & 54.1 & 51.2 & 53.7 & 58 & 34.6 & 32.4 & 35.3 & 35.3 & 34.6 & 35.5 & 36.5 \\
\hline EqcA1 & 62.3 & 56.9 & 63.2 & 64.3 & 41.6 & 40 & 43.2 & 41.8 & 40.9 & 43.1 & 43.4 \\
\hline FecA1 & 54.9 & 51.9 & 59.3 & 60 & 34.3 & 35.4 & 37.2 & 36.8 & 37 & 35.4 & 39.3 \\
\hline HosA1 & 61.8 & 54.9 & 63.5 & 65.3 & 38.6 & 45 & 42.9 & 37.9 & 42.6 & 39.5 & 44.2 \\
\hline BotB & 37.3 & 31.6 & 38.5 & 39.2 & 31.6 & 31.9 & 35.5 & 28.9 & 39.2 & 32.6 & 36.5 \\
\hline CalfB & 38.7 & 35.5 & 40.8 & 39.6 & 33.1 & 33.3 & 38.9 & 33.3 & 39.9 & 35.7 & 39.8 \\
\hline FecB & 41.5 & 37.8 & 41.7 & 41.4 & 30.7 & 31 & 38.9 & 36.5 & 40.3 & 38 & 40.8 \\
\hline EqcB & 39.9 & 38.3 & 43.5 & 43.1 & 28.9 & 32.8 & 36.9 & 36.5 & 40.6 & 37.1 & 38.3 \\
\hline HosB & 43.3 & 33.7 & 40.1 & 41 & 30.4 & 28.8 & 39.9 & 36.9 & 41.5 & 39.9 & 40.2 \\
\hline EqcD1 & 50.2 & 44.8 & 51.6 & 48.2 & 62.3 & 64.3 & 39.3 & 34.1 & 39.1 & 34.1 & 40.1 \\
\hline HosE & 41.2 & 37.1 & 40.3 & 40.6 & 36.7 & 36.1 & 79.4 & 36.2 & 83.5 & 34.6 & 76.9 \\
\hline EqcW1 & - & 61.7 & 69 & 69.6 & 41 & 41.3 & 41.8 & 36.6 & 41.4 & 37.6 & 40.8 \\
\hline FecW1 & 40.1 & - & 66.7 & 70.4 & 36.7 & 35.8 & 38.7 & 33.3 & 37.8 & 31.6 & 37.7 \\
\hline HosW & 33.6 & 34.5 & - & 73.8 & 44 & 43.8 & 41.5 & 39.8 & 40.2 & 39.6 & 40 \\
\hline HosT & 31.7 & 30.1 & 27.3 & - & 44.6 & 38.4 & 43.3 & 37.9 & 41.8 & 38.4 & 44.4 \\
\hline FecDp & 66 & 73.3 & 63.7 & 63 & - & 49.7 & 38.3 & 28 & 36.4 & 28.3 & 38.3 \\
\hline HosD & 70.1 & 80.2 & 60.9 & 72.6 & 36.3 & - & 31.5 & 31.7 & 31.9 & 28.1 & 32.9 \\
\hline CalfE & 78.8 & 96.1 & 85.3 & 77.9 & 84.2 & 103.9 & - & 39 & 82.6 & 36.7 & 88.3 \\
\hline CalfK & 128.1 & 121.3 & 103 & 121.3 & 135.1 & 142.4 & 99.4 & - & 37.5 & 82.1 & 32.2 \\
\hline EqcE & 74.7 & 93.3 & 82.2 & 77.8 & 84 & 98.6 & 15.4 & 100 & - & 37.1 & 80.9 \\
\hline EqcK & 136.6 & 130 & 103.2 & 117.4 & 127 & 145.3 & 98.9 & 16.8 & 99.5 & - & 32.9 \\
\hline FecE & 81.6 & 96.7 & 92.5 & 76.4 & 80.8 & 108.6 & 11.8 & 108.5 & 17.3 & 101.9 & - \\
\hline
\end{tabular}

Percent similarity in upper triangle and percent divergence in lower triangle 
A

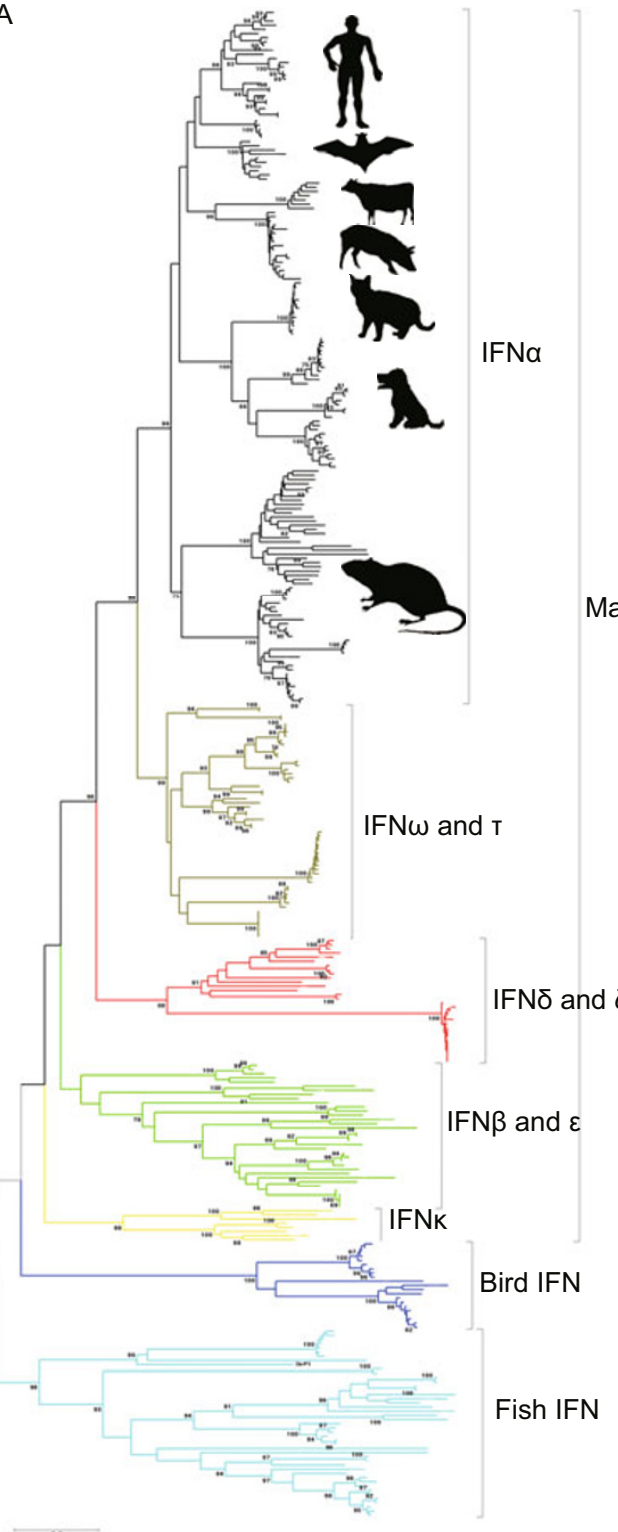

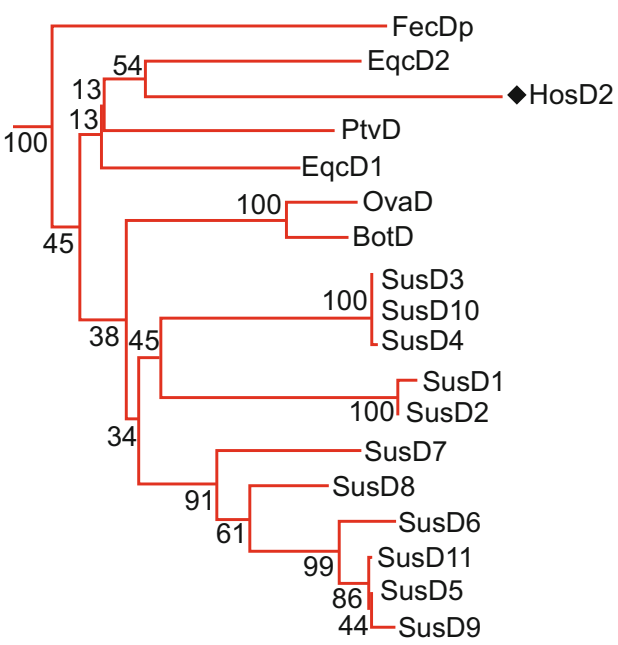

$\mathrm{C}$

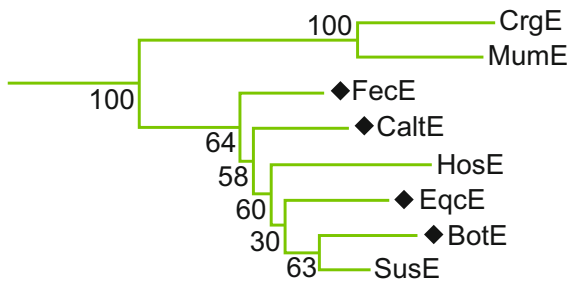

D

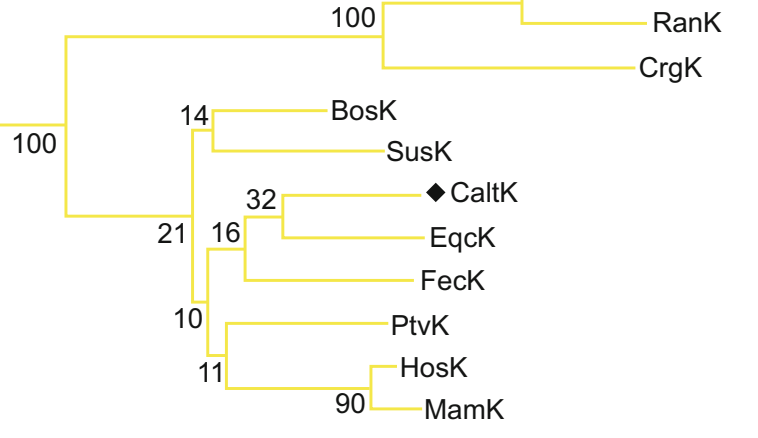

Figure 3. Phylogenetic tree analysis of all type I IFNs including the novel identified IFNs. (A) Overview for the phylogenic tree of type I IFNs. Symbols inside the IFNa clade are human, bat, cow, pig, cat, dog, mouse, respectively. (B) Details for IFNס clades. (C) Details for the IFN $\varepsilon$ clades. (D) Details for the IFNk clades. Bootstrap values over $75 \%$ are shown.

IFNT maybe the last mammalian type I IFN to evolve.

IFNa generally forms species-specific clades in the IFNa subgroup unless the two species in question are closely related. This renders direct homologs of IFNa between different species difficult to find, suggesting a high selection pressure on IFNa during evolution.

\section{Overview of type I IFN evolution}

On the basis of the phylogenetic analysis above and the known characteristics of type I IFNs, we propose a hypothesis for type I IFN evolution: The ancestral type I IFN gene likely contained introns, similar to piscine type I IFNs, and was liable to duplicate which seems to be the ancestor of IFNa genes instead of IFN $\beta$ genes. Synchronously with species evolution, type I IFN genes retain original characteristics in amphibian genomes. IFN $\beta$ evolved from the ancestral IFNa-like genes soon after reptiles arose (Fig. 4). This ancient IFN $\beta$ gene may be encoded by several exons, as no obviously homologs of avian IFN $\beta$ genes can be found in the green lizard genome, and this gene represents the ancestor of avian and mammalian IFN $\beta$ genes, as avian type I IFNs are homolog of mammalian type I IFNs (Sick et al., 1998). Consistent with species evolution from 


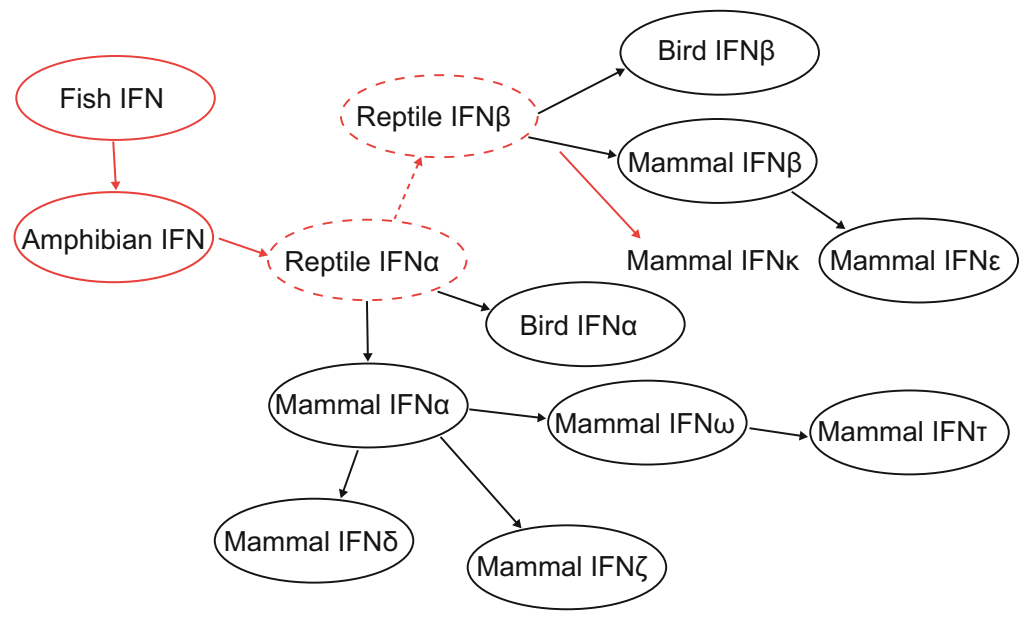

Figure 4. Evolution history of type I IFNs. The type IIFN genes of fish and Amphibian contain 4-5 introns. No reptile IFN genes were cloned as recent studies, but it is no doubt that the IFNa genes do exist as the completeness of the revolution routes. The red circle donates a gene with introns, and dotted line represents a gene or a route which is inferred.

reptiles to birds and mammals, a retrotransposition occurred in the type I IFNs other than IFNK, as evident by the fact that most bird and mammal type I IFNs are intronless. IFNk genes may have arisen from a putative reptile IFN $\beta$ gene before this retrotransposition occurred, and avian type I IFNs remain in their ancestral state. In contrast, mammals encountered more evolutionary pressure to survive in tougher environments, and their type I IFNs divided into many subtypes.

IFN $\beta$ genes duplicated, and one of them represented the ancestor of mammalian IFN $\varepsilon$. This subtype of type I IFNs likely arose at the very beginning of mammalian evolution as practically every mammal possess IFN $\beta$ and IFN $\varepsilon$. Afterward, IFN $\beta$, together with IFN $\varepsilon$ and IFNK, remained untouched as duplications of these genes seem to be difficult (only platypus and cow have two IFN $\beta$-like genes) for unknown reasons.

As avian IFNa and mammalian IFNa genes are strict homologues of the ancient IFNa-like genes and IFNa genes show broader distribution than the other multigene Type I IFNs, the complex mammalian Type I IFN system should be shaped by ancient mammalian IFNa genes. In addition, mammalian IFNa exhibits common activities compared with the other mammalian IFNs. In contrast to IFN $\beta$, it is much easier to duplicate IFNa as approximately 10 IFNa genes are present in most vertebrates. With selective pressure, some of these genes gained specific functions. One of these genes became the forerunner of IFN $\omega$ and duplicated into a cluster of genes. Afterward, a few of the IFN $\omega$ genes in the ruminant ancestor diverged into IFNT to help form placentas. One of the ancient IFNa genes evolved into the forerunner of the IFNס and IFNל genes. The divergence of IFN $\delta$ and IFN $\zeta$ is likely different results of the same selection pressure on different species. During this selection, IFNס gained a reproductive response element in the porcine and ovine genomes, while it failed in the mouse genome.
Synteny alignment of the mouse, dog, pig, and cow type I IFN clusters against the human type I IFN cluster

The type I IFN cluster is not strictly conserved in all mammal genomes, especially itsintergenic regions. Part of the limitin genic regions displays limited similarity with human IFNa, and all of the limitin genes are located together in the type I IFN cluster (Fig. 5).This indicates that the master force for limitin gene family conformation was duplication instead of conversion, and this kind of duplication occurred after the speciation of mice. All mouse IFNa genes have homologous regions in the human genome, and most of the upstream region of these genes is conserved, ensuring that IFNa can be induced by viral infection and pathological processes. The corresponding region of human IFN $\omega$ in the mouse genome seems to be mouse IFNa14, which is evidence for the hypothesis that IFN $\omega$ evolved from IFNa.

IFNT cannot be identified in the human type I IFN cluster, and only a previously described pseudogeneis located outside of the gene cluster on the IFN $\beta$ side. However, a discontinuous region of the human genome displays limited conservation with IFNT genes, and further analysis revealed that these regions are microRNAs, such as the MIR31 host gene.

\section{DISCUSSION}

In the present study, an IFNס-like gene in the human genome was identified, refuting the concept that humans have no IFNס genes (Pestka et al., 2004). This gene is located on chromosome 9 , with a pseudogene named IFNA12p inside of it. Both phylogenic and sequence similarity analyses demonstrated that this gene is an IFNס gene. In the phylogenic tree, the mammalian IFN $\beta$, IFN $\varepsilon$, and IFNk genes formed a clade separate from the other mammalian type I IFNs, while piscine and avian IFNs formed distinct clades. Based on our phylogenetic 
A

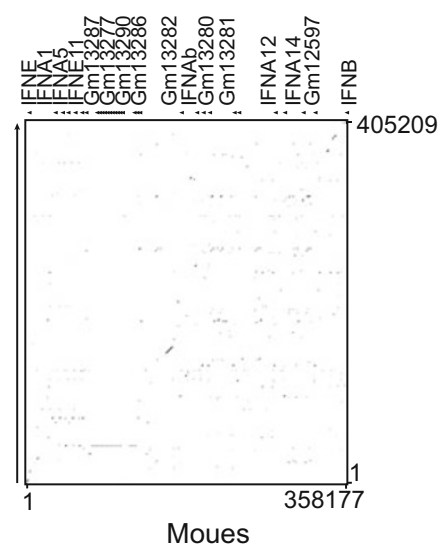

D

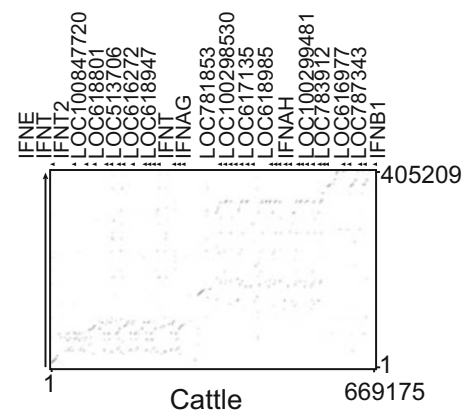

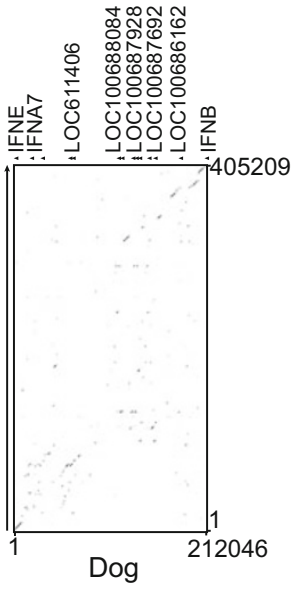

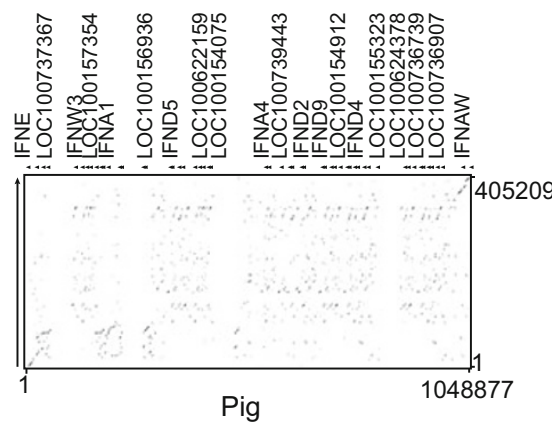

Figure 5. Dot-plot analysis reveals a complex pathway of type I IFN evolution. Dotplot analysis was applied to the genic and intergenic regions of the mouse (A), dog (B), pig (C) and cattle (D) type I IFN gene clusters to human type I IFN cluster. The type I IFN genes are cited as NCBI shows. IFN-like proteins are also cited. The alignment shows as intermittent lines which represent the regions with at least $70 \%$ sequence identity, indicating that the type I IFN cluster is not strictly conserved in all mammal genomes, especially its intergenic regions.

analysis and the known characteristics of the various type I IFNs, we hypothesized the evolutionary history of type I IFNs. In this evolution route, IFNa formed a core from which new IFNs divided during vertebrate evolution. One of the IFNa genes evolved away from the other genes and became the ancestor of IFN $\beta$ in reptiles, and most of these genes lost introns through retrotranspositions when birds and mammals diverged from reptiles. The ancestor IFNa-like genes duplicated more frequently than the ancestor IFN $\beta$-like gene and divided into several subtypes (including IFNס, IFNT, and IFNל) during the evolution of some animals, while IFNßevolved into IFN $\varepsilon$ and IFNK at the very beginning of the emergence of mammals.

The doubts and suspicions in this evolutionary model focus on the time when the type I IFN introns disappeared and whether reptiles genomes encode IFN $\beta$ genes. Type I IFNs exist in all kinds of vertebrates, but only the fish and amphibian type I IFN genes contain four introns as recent research (Qi et al., 2010). In the fish genome, IFNa, IFNb, IFNc and IFNd are considered to be different kinds of type I IFNs that only exists in fish and not strictly orthology of higher vertebrates IFN genes. Both IFNa, IFNb, IFNc and IFNd displays a considerable distance to the other type I IFNs in our phylogenetic tree, but IFNa and IFNc show similar antiviral activities and ability to induce antiviral genes, like mammalian IFNa do, while IFNb and IFNd show little activities (Sun et al., 2009; Svingerud et al., 2012).

When the introns of these type I IFN genes disappeared remains unknown, but the IFN genes in amphibians may have introns (Qi et al., 2010). It is very likely that reptile genomes encode IFNs for the sake of the completeness of evolution routes, even though no reptiles IFN gene has yet been cloned, only been inferred. The failure to detect reptile IFNs when using chicken IFNa genes with BLAST against the green lizard genome suggests that IFN genes in reptiles are much similar to fish IFN genes than those in warm-blooded animals. However, the IFNk genes of mammals have one intron each, suggesting that the existence of intron-containing reptile IFN $\beta$ genes exist.

Amphibian IFNs represent as intron-containing IFNs with five different molecules as inferred by present research, however, functional study on frog type I IFNs is still needed (Qi et al., 2010). A retrotransposition event likely occurred to type I IFNs during reptile evolution as avian and mammalian type I IFNs are intronless genes. Type I IFN genes in lizards may contain introns as no obvious homologs of avian and mammalian type I IFN genes can be found in the green lizard genome. The situation in snakes and turtles remains unknown, and perhaps intron-containing and intronless type I IFN genes coexist in their genomes. IFN $\beta$ genes likely exist in reptile genomes because these genes appear to exist in all avian and mammalian genomes. The IFN $\beta$ gene may have duplicated during reptile evolution into mammals, and retrotransposition occurred simultaneously. Further, the IFNk genes in mammals each contain an intron, and IFN $\varepsilon$ genes are intronless, suggesting the possibility that duplicated IFN $\beta$ genes or the ancestor of 
IFNk genes exist in reptilian genomes.

Even though all type I IFNs bind to the same receptors, different IFN display different bioactivities. The functions of type I IFN maintain good relation with their evolutions. The antiviral activity and anti-proliferation activity of IFN $\beta$ is lower than most of the IFNas, and the antiviral activity of IFNa-evoluted IFNs is stronger than that of IFNK and IFNعs (Sang et al., 2010; Lavoie et al., 2011). The ISGs that IFN $\beta$ induces tend to regulate host immunoreactions, while IFNa tends to induce antiviral proteins, such as 2'-5' OAS and PKR (Qu et al., 2013). This may be caused by the different ways that they interact with IFN receptor subunits. Despite their differences in sequence, IFNwdemonstrates little difference with IFNa in their expression, function, ternary complex structures with receptors, and abilities to induce ISGs (Thomas et al., 2011). Expressed by trophoblasts before they attach to the placenta, IFNT is important to prevent the corpus luteum from degradation and, hence, ensuring the pregnancy continues, while IFNס is expressed in conceptus and shows a potent ability to regulate pregnancy (Chelmońskasoyta, 2002; Roberts, 2007).It has been hypothesized that IFNT is not virally inducible and that its function has no relationship to pathogenesis, which is quite different from the other multigene Type I IFNs, but recent research shows that bovine IFNTis an antiviral protein capable of inducing 2'$5^{\prime}$ OAS with less toxicity to the cells, which suggests that IFNT could be a better drug than IFNa for patients suffering from viruses(Johnson et al., 2001). The IFN $\beta$-evoluted IFNs, IFN $\varepsilon$ and IFNK, tends to induce effectors which can regulate host immunoreactions. Over expression of IFNk in pancreatic islets can induce diabetes in mouse, and recent research has shown that the expression of IFNK in skin has a close association with systemic lupus erythematosus and inflammation (Vassileva et al., 2003; Harley et al., 2010). IFN $\varepsilon$ can enhance the lymphocyte recruitment to lung alveoli with reduced inflammation, promote migration of antigen-specific CD8+ T cells to the gut, which suggests IFN $\varepsilon$ an important role in mucosal immunity (Xi et al., 2012). This relationship between evolution and function of type I IFN may provide a new view for type I IFN function analysis.

\section{MATERIALS AND METHODS}

Genome selection

A total of 496 IFN sequences from 99 species were acquired from $\mathrm{NCBI}($ http://www.ncbi.nlm.nih.gov/). Allelic genes and pseudogenes were omitted for the sake of phylogenetic analyses. The IFN genes from human, mouse, and pig have already been characterized in detail before, and some of the features of IFNT genes from ruminants and IFNס genes from pigs have also already been described (Bazer et al., 1997). To find undetected IFN genes, BLASTn (Buhler et al., 2007) was used to search 13 genomes (human, mouse, dog, cat, sheep, pig, cow, horse, chicken, zebrafish, green anole, Nile tilapia, and western clawed frog). Human IFNa2b (AY255838.1), IFN IFN $\beta$ (M28622.1), IFN $\varepsilon$ (AY190045.1), and IFNK (AF315688.1), mouse IFNZ1 (NM_197889.2), pig IFNס1 (GQ415074.1), and cow
IFNT1 (AF238611.1), chicken IFNa1 (AB021153.1) and chicken IFN $\beta$ (AY974089.1) were chosen as initial queries. All of the default alignment/search parameters were used. Sequences displaying $>10 \%$ similarity were collected for further identification. Short homologous sequences were extended to $600 \mathrm{bp}$ to calculate the similarity with given sequences.

\section{Phylogenetic analyses}

All of the IFN sequences were aligned with ClustalW (EBI, www.ebi. ac.uk/clustalw) (Thompson et al., 2002). After manual correction of the sequences, a neighbor-joiningtree was constructed using MAGE 5.0 with the following parameters:method $=\mathrm{NJ}$, substitution model $=$ Poisson correction method, and 1000 bootstrap replicates (Tamura et al., 2011). We also constructed a maximum likelihood tree with the following parameters to evaluate the reproducibility of the grouping of the IFN genes:method JTT and 1000 bootstrap replicates.

\section{Gene synteny analysis}

MultiPipmaker was used to align both the genic and intergenic regions of the mouse, dog, pig, and cow type I IFN gene clusters to the human type I IFN cluster (Schwartz et al., 2003). Default parameters were used except that the "search one strand" and "single coverage" options were chosen. Genes were cited as shown by NCBI.

Competing Interests: The authors have declared that no competing interests exist.

\section{ACKNOWLEDGEMENTS}

This study was funded by the National Natural Science Foundation of China (Grant No. 31100644), the Ministry of Science and Technology program of China (Grant Nos. 2011AA10A215, and 2010GB24910698). Wenjun Liu is a principal investigator of the National Natural Science Foundation of China Innovative Research Group (Grant No. 81021003). The funders had no role in study design, data collection and analysis, decision to publish, or preparation of the manuscript.

\section{ABBREVIATIONS}

Bot, Bostaurus Cow; Crg, Cricetulusgriseus Hamster; Calf, Canis lupus familiaris Dog; Eqc, Equuscaballus Horse; Fec, Feliscatus Cat; Hos, Homo sapiens Human; ISG, interferon-stimulated gene; ISGF, interferon-stimulated gene factor; Mum, Musmusculus Mouse; Ova, Ovisaries Sheep; Ptv, Pteropus vampyrus Bat; Ran, Rattusnorvegicus Rat; Sus, Susscrofa Pig

\section{COMPLIANCE WITH ETHICS GUIDELINES}

Xu Lei, Yang Limin, and Liu Wenjun declare that they have no conflict of interest.

This article does not contain any studies with human or animal subjects performed by the any of the authors.

\section{REFERENCES}

Altmann, S.M., Mellon, M.T., Distel, D.L., and Kim, C.H. (2003). Molecular and functional analysis of an interferon gene from the zebrafish, Danio rerio. J Virol 77, 1992-2002. 
Bazer, F.W., Spencer, T.E., and Ott, T.L. (1997). Interferon tau: A novel pregnancy eecognition signal. Am J Reprod Immunol 37, 412-420.

Buhler, J.D., Lancaster, J.M., Jacob, A.C., and Chamberlain, R.D. (2007). Mercury BLASTN: Faster DNA sequence comparison using a streaming hardware architecture. In Reconfigurable Systems Summer Institute, Oxford University Press, Oxford, 1-8.

Chelmońskasoyta, A. (2002). Interferon tau and its immunobiological role in ruminant reproduction. Arch Immunol Ther Exp 50, 47-52.

Cochet, M., Vaiman, D., and Lefèvre, F. (2009). Novel interferon delta genes in mammals: Cloning of one gene from the sheep, two genes expressed by the horse conceptus and discovery of related sequences in several taxa by genomic database screening. Gene 433, 88-99.

David, M. (2002). Signal transduction by type I interferons. Biotechniques (Suppl), 58-65.

Hardy, M.P., Owczarek, C.M., Jermiin, L.S., Ejdebäck, M., and Hertzog, P.J. (2004). Characterization of the type I interferon locus and identification of novel genes. Genomics 84, 331-345.

Harley, I.T.W., Niewold, T.B., Stormont, R.M., Kaufman, K.M., Glenn, S.B., Franek, B.S., Kelly, J.A., Johnson, G.A., Stewart, M.D., Gray, C.A., Choi, Y., Burghardt, R.C., Yu-Lee, L.Y., Bazer, F.W., and Spencer, T.E. (2001). Effects of the estrous cycle, pregnancy, and interferon tau on 2',5'-oligoadenylate synthetase expression in the ovine uterus. Biol Repr 64, 1392-1399.

Henco, K., Brosius, J., Fujisawa, A., Fujisawa, J.I., Haynes, J.R., Hochstadt, J., Kovacic, T., Pasek, M., Schambock, A., Schmid, J., et al. (1985). Structural relationship of human interferon alpha genes and pseudogenes. J Mol Biol 185, 227-260.

Kilpatrick, J.R., Hutchings, D., Divers, J., et al. (2010). The role of genetic variation near interferon-kappa in systemic lupus erythematosus. J Biomed Biotechnol 2010, 1-11.

Isaacs, A., and Lindenmann, J. (1957). Virus interference. I. The interferon. Proc Roy Soc B: Biol Sci 147, 258-267.

Lavoie, T.B., Kalie, E., Crisafulli-Cabatu, S., Abramovich, R., DiGioia, G., Moolchan, K., Pestka, S., and Schreiber, G. (2011). Binding and activity of all human alpha interferon subtypes. Cytokine 56, 282-289.

Lefèvre, F., and Boulay, V. (1993). A novel and atypical type one interferon gene expressed by trophoblast during early pregnancy. J Biol Chem 268, 19760-19768.

Oritani, K., Medina, K.L., Tomiyama, Y., Ishikawa, J., Okajima, Y., Ogawa, M., Yokota, T., Aoyama, K., Takahashi, I., Kincade, P.W., et al. (2000). Limitin: An interferon-like cytokine that preferentially influences B-lymphocyte precursors. Nat Med 6, 659-666.

Pestka, S., Krause, C.D., and Walter, M.R. (2004). Interferons, interferon-like cytokines, and their receptors. Immunol Rev 202, 8-32.

Qi, Z.T., Nie, P., Secombes, C.J., and Zou, J. (2010). Intron-containing type I and type III IFN coexist in amphibians: Refuting the concept that a retroposition event gave rise to type I IFNs. J Immunol 184, 5038-5046.

Qu, H., Yang, L., Meng, S., Xu, L., Bi, Y., Jia, X., Li, J., Sun, L., and Liu, W. (2013). The differential antiviral activities of chicken interferon $\alpha$ (ChIFN- $\alpha$ ) and ChIFN- $\beta$ are related to distinct interferon-stimulated gene expression. PLoS ONE 8, e59307.

Roberts, R.M., Liu, L., Guo, Q.T., Leaman, D., and Bixby, J. (1998). The evolution of the type I interferons. J Interferon Cytokine Res 18, 805-816.
Roberts, R.M. (2007). Interferon-tau, a type 1 interferon involved in maternal recognition of pregnancy. Cytokine Growth Factor Rev 18, 403-408.

Robertsen, B., Bergan, V., Røkenes, T., Larsen, R., and Albuquerque, A. (2003). Atlantic salmon interferon genes: cloning, sequence analysis, expression, and biological activity. J Interferon Cytokine Res 23, 601-612.

Sang, Y., Rowland, R.R., Hesse, R.A., and Blecha, F. (2010). Differential expression and activity of the porcine type I interferon family. Physiol Genomics 42, 248-258.

Schwartz, S., Elnitski, L., Li, M., Weirauch, M., Riemer, C., Smit, A., Program, N.C.S., Green, E.D., Hardison, R.C., and Miller, W. (2003). MultiPipMaker and supporting tools: alignments and analysis of multiple genomic DNA sequences. Nucleic Acids Res 31, 3518-3524.

Sheppard, P., Kindsvogel, W., Xu, W., Henderson, K., Schlutsmeyer, S., Whitmore, T.E., Kuestner, R., Garrigues, U., Birks, C., Roraback, J., et al. (2003). IL-28, IL-29 and their class II cytokine receptor IL28R. Nat Immunol 4, 63-68.

Sick, C., Schultz, U., Münster, U., Meier, J., Kaspers, B., and Staeheli, P. (1998). Promoter structures and differential responses to viral and nonviral inducers of chicken type I interferon genes. J Biol Chem 273, 9749-9754.

Sick, C., Schultz, U., and Staeheli, P. (1996). A family of genes coding for two serologically distinct chicken interferons. J Biol Chem 271, 7635-7639.

Stancato, L.F., Sakatsume, M., David, M., Dent, P., Dong, F., Petricoin, E.F., Krolewski, J.J., Silvennoinen, O., Saharinen, P., et al. (1997). Beta interferon and oncostatin $\mathrm{M}$ activate Raf-1 and mitogen-activated protein kinase through a JAK1-dependent pathway. Mol Cell Biol 17, 3833-3840.

Stark, G.R., Kerr, I.M., Williams, B.R.G., Silverman, R.H., and Schreiber, R.D. (1998). How cells respond to interferons. Annu Rev Biochem 67, 227-264.

Sun, B.J., Robertsen, B., Wang, Z.Q., and Liu, B. (2009). Identification of an Atlantic salmon IFN multigene cluster encoding three IFN subtypes with very different expression properties. Dev Comp Immunol 33, 547-558.

Svingerud, T., Solstad, T., Sun, B.J., Nyrud, M.L.J., Kileng, Ø., GreinerTollersrud, L., and Robertsen, B. (2012). Atlantic salmon type I IFN subtypes show differences in antiviral activity and cell-dependent expression: Evidence for high IFNb/IFNc-producing cells in fish lymphoid tissues. J Immunol 189, 5912-5923.

Tamura, K., Peterson, D., Peterson, N., Stecher, G., Nei, M., and Kumar, S. (2011). MEGA5: Molecular evolutionary genetics analysis using maximum likelihood, evolutionary distance, and maximum parsimony methods. Mol Biol Evol 28, 2731-2739.

Thomas, C., Moraga, I., Levin, D., Krutzik, P.O., Podoplelova, Y., Trejo, A., Lee, C., Yarden, G., Vleck, S.E., Glenn, J.S., et al. (2011). Structural linkage between ligand discrimination and receptor activation by type I interferons. Cell 146, 621-632.

Thompson, J.D., Gibson, T.J., and Higgins, D.G. (2002). Multiple sequence alignment using ClustalW and ClustalX. In Current Protocols in Bioinformatics, John Wiley \& Sons, Inc.

Vassileva, G., Chen, S.C., Zeng, M., Abbondanzo, S., Jensen, K., Gorman, D., Baroudy, B.M., Jiang, Y., Murgolo, N., and Lira, S.A. (2003). Expression of a novel murine type I IFN in the pancreatic islets 
induces diabetes in mice. J Immunol 170, 5748-5755.

Whaley, A.E., Meka, C.S., Harbison, L.A., Hunt, J.S., and Imakawa, K. (1994). Identification and cellular localization of unique interferon mRNA from human placenta. J Biol Chem 269, 10864-10868.

Woelk, C.H., Frost, S.D.W., Richman, D.D., Higley, P.E., and Kosako- vsky Pond, S.L. (2007). Evolution of the interferon alpha gene family in eutherian mammals. Gene 397, 38-50.

Xi, Y., Day, S.L., Jackson, R.J., and Ranasinghe, C. (2012). Role of novel type I interferon epsilon in viral infection and mucosal immunity. Mucosal Immunol 5, 610-622. 